Check for updates

Cite this: Soft Matter, 2018, 14,5922

Received 10th April 2018, Accepted 18th June 2018

DOI: $10.1039 / c 8 s m 00739 j$

rsc.li/soft-matter-journal

\section{Ideal wet two-dimensional foams and emulsions with finite contact angle $\uparrow$}

\author{
S. J. Cox, (D)*a A. M. Kraynik, ${ }^{\text {bc }}$ D. Weaire ${ }^{c}$ and S. Hutzler (D) ${ }^{c}$
}

\begin{abstract}
We present simulations that show that the equilibrium structure of an ideal two-dimensional foam with a finite contact angle develops an inhomogeneity for high liquid fraction $\phi$. In liquid-liquid emulsions this inhomogeneity is known as flocculation. In the case of an ordered foam this requires a perturbation, but in a disordered foam inhomogeneity grows steadily and spontaneously with $\phi$, as demonstrated in our simulations performed with the Surface Evolver.
\end{abstract}

\section{Introduction}

In emulsions, the term flocculation refers to the (spontaneous) clustering of droplets, leading to the formation of density inhomogeneities. Here we describe the onset of flocculation in computer simulations of two-dimensional (2D) liquid foams. This only occurs in systems where the liquid-gas interfaces meet at a finite contact angle $\theta$, as illustrated in Fig. 1(b). Previous simulations of $2 \mathrm{D}$ foams with finite liquid fraction $\phi$ have taken this contact angle to be zero ${ }^{1}$ or, where that was not feasible for numerical reasons, as small as possible. ${ }^{2}$ We treat both liquid and gas as incompressible, since the pressure differences between bubbles are much smaller than atmospheric pressure, and so the results apply equally to emulsions.

Two-dimensional foams have properties that are broadly similar to their three-dimensional counterparts, but are much simpler to analyse. Their study has early antecedents, ${ }^{3}$ and continues to be of interest today. The usual theoretical model is entirely two-dimensional, while the third dimension may be significant in relevant experimental systems, such as a foam trapped between two plates. ${ }^{3,4}$

A simulated example of an ideal 2D foam with finite liquid fraction $\phi$ and finite contact angle $\theta$ is shown in Fig. 1(a). It was produced by the method described in Appendix A, which minimizes the surface energy of the foam. The gas bubbles are surrounded by a network of smoothly-curved thin films connecting the liquid-filled Plateau borders, which each have three or more sides. There are therefore two types of interface:

\footnotetext{
${ }^{a}$ Department of Mathematics, Aberystwyth University, Aberystwyth SY23 3BZ, UK. E-mail:foams@aber.ac.uk

${ }^{b}$ Sandia National Laboratories (retired), Albuquerque, New Mexico, USA

${ }^{c}$ School of Physics, Trinity College Dublin, The University of Dublin, Ireland

$\dagger$ Electronic supplementary information (ESI) available. See DOI: 10.1039/c8sm00739j
}

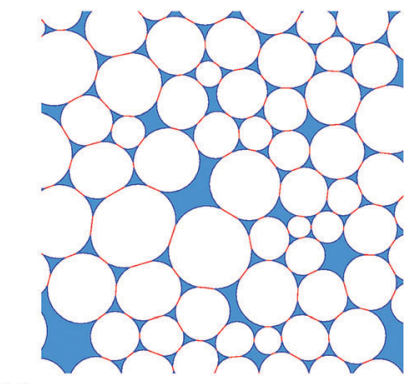

(a)

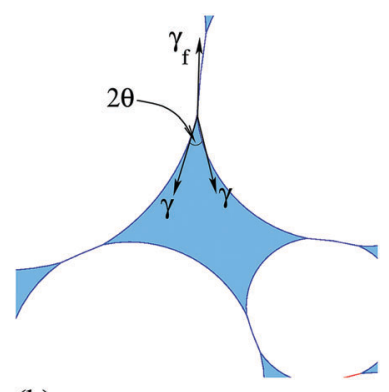

(b)

Fig. 1 (a) Typical simulation of a two-dimensional foam with liquid fraction $\phi=0.1$ and contact angle $\theta=9.5^{\circ}$. The image shows part of an equilibrated foam of 1500 bubbles. (b) Close-up of a four-sided Plateau border, showing the contact angle $\theta$. The vectors represent the forces, due to interfacial tension, acting on the point of contact.

liquid-gas interfaces around each Plateau border, and gasliquid-gas interfaces forming the bubble-bubble contacts. On each of the interfaces the Laplace-Young law relates the product of interfacial tension and curvature to the pressure difference across the interface, ${ }^{5,6}$ and consequently liquid films are represented by arcs of circles that meet at the vertices of Plateau borders. The films are considered to be infinitesimally thin, so all of the liquid in the foam is considered to be contained in the Plateau borders.

A finite contact angle implies that the interfacial tension associated with the bubble-bubble interfaces is less than twice that associated with the Plateau borders (see Fig. 1b). Note that throughout this paper "interfacial tension" is used for what is really a line tension (or energy per unit line length) in such an idealised 2D model. The contact angle $\theta$ is given by

$$
\theta=\cos ^{-1}\left(\frac{\gamma_{\mathrm{f}}}{2 \gamma}\right)
$$




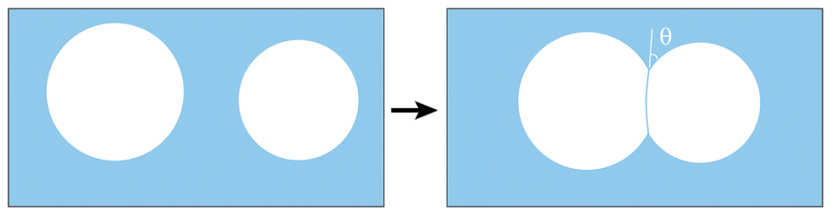

Fig. 2 The (line) energy of two isolated circular bubbles is reduced when they share a common interface, if the contact angle $\theta$ is finite.

where $\gamma_{\mathrm{f}}$ is the interfacial tension of a liquid film; this is equal to or smaller than twice the (bulk) interfacial tension $\gamma$ associated with the gas-liquid interfaces of a Plateau border. ${ }^{7}$ More precisely, writing $\ell$ for the film thickness, the film tension is given by

$$
\gamma_{\mathrm{f}}=2 \gamma+V(\ell)
$$

where $V(\ell)$ is an effective interface potential. $V$ can estimated from DLVO theory: it is negative and its derivative is equal to minus the disjoining pressure. ${ }^{8}$ The surface energy of the foam is the sum of the lengths of all interfaces, multiplied by their appropriate interfacial tension.

For bubbles immersed in a liquid, the presence of finite contact angles entails net attractive forces between them (see Fig. 2) when they are only slightly compressed together. This is similar to the attraction between droplets, when considering emulsions. $^{9}$

In the present paper we address some basic consequences of introducing finite contact angles into the standard model of $2 \mathrm{D}$ foams, by analysing simulations carried out with the Surface Evolver software of Ken Brakke. ${ }^{10}$ We will see that finite contact angles, even if apparently very small, can have large effects.

Henry Princen introduced the concept of a contact angle between a thin liquid film and its adjacent Plateau border ${ }^{11}$ and he established its physical significance in foams and emulsions by analysing 2D ordered (hexagonal) monodisperse structures $^{7}$ which admit analytical solutions. The Surface Evolver and current computational resources enable the simulation of disordered foams, which is the usual practical case of interest. Princen's work was stimulated by his own measurements of contact angles for soap films in contact with bulk solution. ${ }^{12} \mathrm{He}$ found that finite contact angles up to $17^{\circ}$ could be achieved in surfactant (SDS) solutions at sufficiently high concentrations of added electrolytes. More recently, contact angles up to $30^{\circ}$ have been found in emulsions. ${ }^{13}$

We shall begin in Section 2 by recapitulating Princen's 2D model of an ordered foam, which gave a number of exact results. This turns out to be instructive when discussing our own results from simulations of disordered polydisperse foams, which are described in Section 3. We summarize our conclusions in Section 4.

\section{Ordered hexagonal foams}

Even the apparently trivial case of an ordered $2 \mathrm{D}$ foam proves to present some challenges to detailed understanding, so we shall examine it carefully.

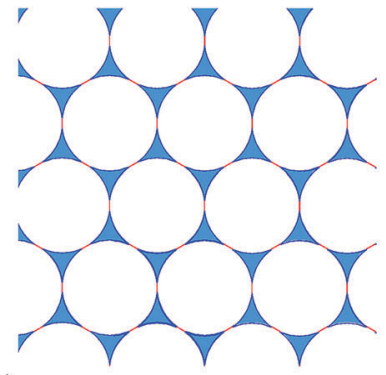

(a)

Fig. 3 Examples of monodisperse hexagonal foams for contact angle $\theta=6.26^{\circ}$ and different values of liquid fraction. (a) Liquid fraction $\phi_{0}^{\theta}=0.084$, where the energy is minimal (state of zero compression, eqn (3)). (b) Liquid fraction $\phi_{m}^{\theta}=0.128$, the maximum value of liquid fraction at which the bubbles still remain in contact (eqn (7)).

Monodisperse 2D bubbles are arranged in equilibrium on an hexagonal lattice (Fig. 3). Princen ${ }^{7}$ showed that the liquid fraction $\phi_{0}^{\theta}$ of such a packing at zero compression, which corresponds to zero osmotic pressure ${ }^{7,14,15}$ and minimal surface energy, ${ }^{7}$ is given by

$$
\phi_{0}^{\theta}=1-\frac{\pi-6 \theta+3 \sin (2 \theta)}{2 \sqrt{3} \cos ^{2} \theta} .
$$

In the case of a zero contact angle $(\theta=0), \phi_{0}^{\theta}$ reduces to the familiar value

$$
\phi_{0}:=\phi_{0}^{\theta=0}=1-\pi /(2 \sqrt{3}) \simeq 0.093,
$$

which is the liquid fraction of a hexagonal close packing of circular bubbles; it is known as the wet limit of an ordered monodisperse foam with zero contact angle. However, the bubbles are never circular when the contact angle is finite. Fig. 3 shows two examples of an ordered foam with finite contact angle, for different values of the liquid fraction.

Princen calculated the work per unit area, $\Delta W^{\theta}$, required to compress (by removing liquid) a foam from the liquid fraction $\phi_{0}^{\theta}$ to any given $\phi,{ }^{7}$ at constant bubble area. The bubble area is written in terms of the radius $R$ of an undeformed circular bubble of the same area, resulting in $\neq$

$$
\begin{aligned}
\frac{\Delta W^{\theta}}{\gamma / R}= & 2\left(1-\phi_{0}^{\theta}\right) \cos \theta \\
& \times\left[\frac{1}{(1-\phi)^{1 / 2}\left(1-\phi_{0}\right)^{1 / 2}}-\left(\frac{\phi_{0}^{\theta}}{1-\phi_{0}}\right)^{1 / 2}\right. \\
& \left.\times\left(\frac{\phi}{1-\phi}\right)^{1 / 2}-\left(\frac{1-\phi_{0}^{\theta}}{1-\phi_{0}}\right)^{1 / 2}\right],
\end{aligned}
$$

where the normalizing factor $\gamma / R$ is often called the Laplace pressure.

\$ Princen ${ }^{7}$ describes his calculation in terms of the deformation of columns of hypothetical cylindrical emulsion drops. We have re-written his expression in terms of liquid fraction $\phi$, rather than gas fraction $(1-\phi)$, and corrected one misprint (missing superscript $\theta$ in his eqn (35)). 


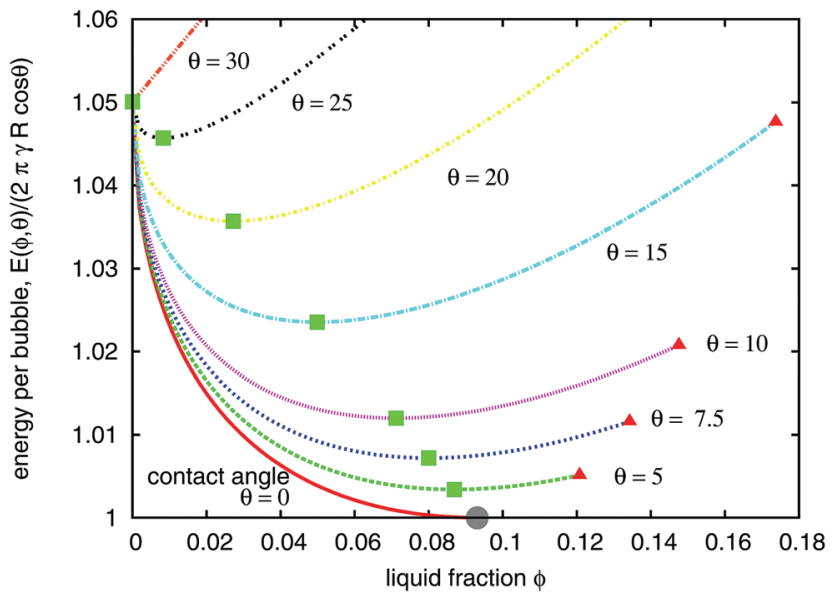

Fig. 4 Variation of the energy per bubble $E(\phi, \theta) /(2 \pi R \gamma \cos \theta)$ (eqn (6)) of an ordered hexagonal 2D foam with liquid fraction $\phi$, for a range of different contact angles $\theta$. ( $\gamma$ : bulk interfacial tension, $R$ : radius of an undeformed (circular) bubble.) Symbols correspond to the critical liquid fractions $\phi_{0}^{\theta}$ (green squares), $\phi_{m}^{\theta}$ (red triangles), and $\phi_{0}$ (grey disc), defined in the text.

In the following we will consider the energy per bubble $E$ as a function of liquid fraction $\phi$ and contact angle $\theta$. Using eqn (5) and $\Delta W^{\theta}=\frac{1-\phi_{0}^{\theta}}{R^{2} \pi}\left(E(\phi, \theta)-E\left(\phi_{0}^{\theta}, \theta\right)\right)$ we obtain

$$
\frac{E(\phi, \theta)}{2 \pi R \gamma \cos \theta}=\frac{1-\sqrt{\phi_{0}^{\theta} \phi}}{\sqrt{(1-\phi)\left(1-\phi_{0}\right)}} .
$$

In the dry limit ( $\phi=0$, i.e., close-packed hexagons), this reduces to $E(0, \theta) /(2 \pi R \gamma \cos \theta)=\left(1-\phi_{0}\right)^{-1 / 2}$.

The energy $E(\phi, \theta)$ in eqn (6) may be characterised by two different critical values of the liquid fraction, as shown in Fig. 4. These are

- the liquid fraction $\phi_{0}^{\theta}$ (eqn (3)) at which the energy has a minimum;

- the maximum value of liquid fraction $\phi_{m}^{\theta}$ at which the bubbles remain in contact, given by

$$
\phi_{m}^{\theta}=\frac{\sqrt{3}}{8 \sin ^{2} \frac{\varphi}{2}}\left[2 \sin \frac{\varphi}{2}\left(\frac{1}{\sqrt{3}} \sin \frac{\varphi}{2}+\cos \frac{\varphi}{2}\right)-\varphi\right],
$$

with $\varphi=\pi / 3-2 \theta$. This is the hypothetical wet limit of an ordered monodisperse foam with finite contact angle.

Fig. 5 displays the variation of both $\phi_{0}^{\theta}$ and $\phi_{m}^{\theta}$ with contact angle $\theta$. Note that $\theta=0$ is a special case because the critical values coincide, $\phi_{0}^{0}=\phi_{m}^{0}=\phi_{0}=1-\pi /(2 \sqrt{3}) \simeq 0.093$. Fig. 3 shows an ordered foam at each of the critical liquid fractions $\phi_{m}^{\theta}$ and $\phi_{0}^{\theta}$, for contact angle $\theta=6.26^{\circ}$.

When the contact angle is finite, the non-circular bubbles at $\phi_{m}^{\theta}$ contact their neighbours at a point. However, this situation differs from the conventional wet limit for zero contact angle, as we explore below.

All points on the curves for energy as a function of liquid fraction, shown in Fig. 4, correspond to (possibly metastable) equilibrium structures. Since the liquid fraction $\phi_{0}^{\theta}$ corresponds to the minimum energy, any homogeneous structure for which

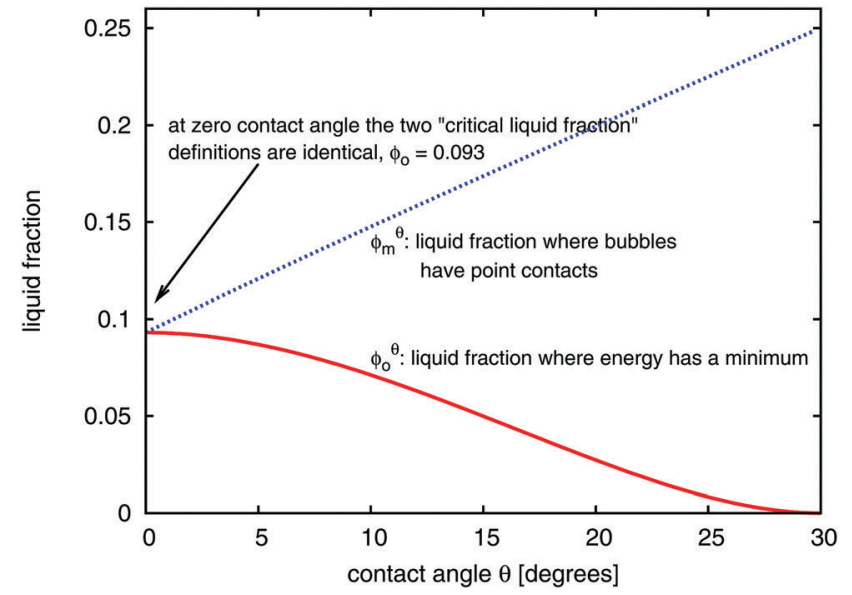

Fig. 5 Variation of the critical liquid fractions $\phi_{0}^{\theta}$ (eqn (3)), and $\phi_{m}^{\theta}$ (eqn (7)) as a function of contact angle $\theta$ in an ordered hexagonal foam.

$\phi>\phi_{0}^{\theta}$ must be metastable, at least for an infinite sample. This is because an inhomogeneous structure can be defined, with constant energy (in the limit of infinite sample size) close to $E\left(\phi_{0}^{\theta}\right)$. This scenario is illustrated in Fig. 6, where we have represented the energy of the inhomogeneous structure by a horizontal line beyond $\phi_{0}^{\theta}$.

The formation of inhomogeneities in the ordered monodisperse structure can be simulated by considering a representative area of foam that contains a large number of bubbles, and increasing the liquid fraction by expanding the system while keeping the bubble areas fixed. An example of such an inhomogeneous structure is shown in Fig. 7. Finite contact angles were included by assigning different values for the line tension in the bubble-bubble interfaces $\left(\gamma_{\mathrm{f}}\right)$ and the Plateau border sides $(\gamma)$; the contact angle is given by eqn (1). (For further details see Appendix A.)

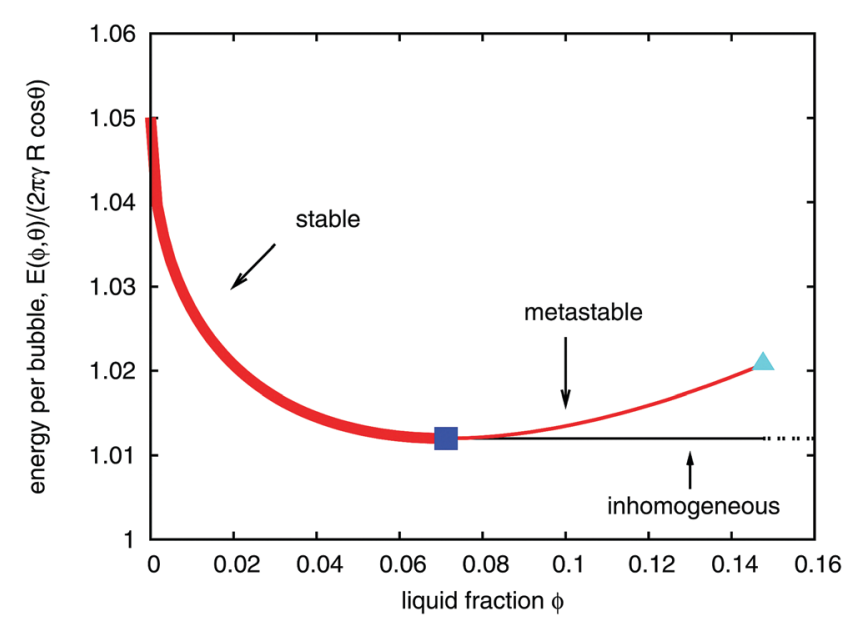

Fig. 6 Variation of the energy per bubble $E(\phi, \theta)$ of an initially ordered hexagonal foam (for contact angle $\theta=10^{\circ}$ ). For values of liquid fraction exceeding $\phi_{0}^{\theta}$ (marked by a blue square), the hexagonal bubble arrangement is metastable, since alternative inhomogeneous structures exist with lower energy. (Fig. 7(a) shows such an example, obtained from simulation.) The light blue triangle at critical liquid fraction $\phi_{m}^{\theta}$ corresponds to the point where bubbles in an hexagonal arrangement no longer touch. 
(a)
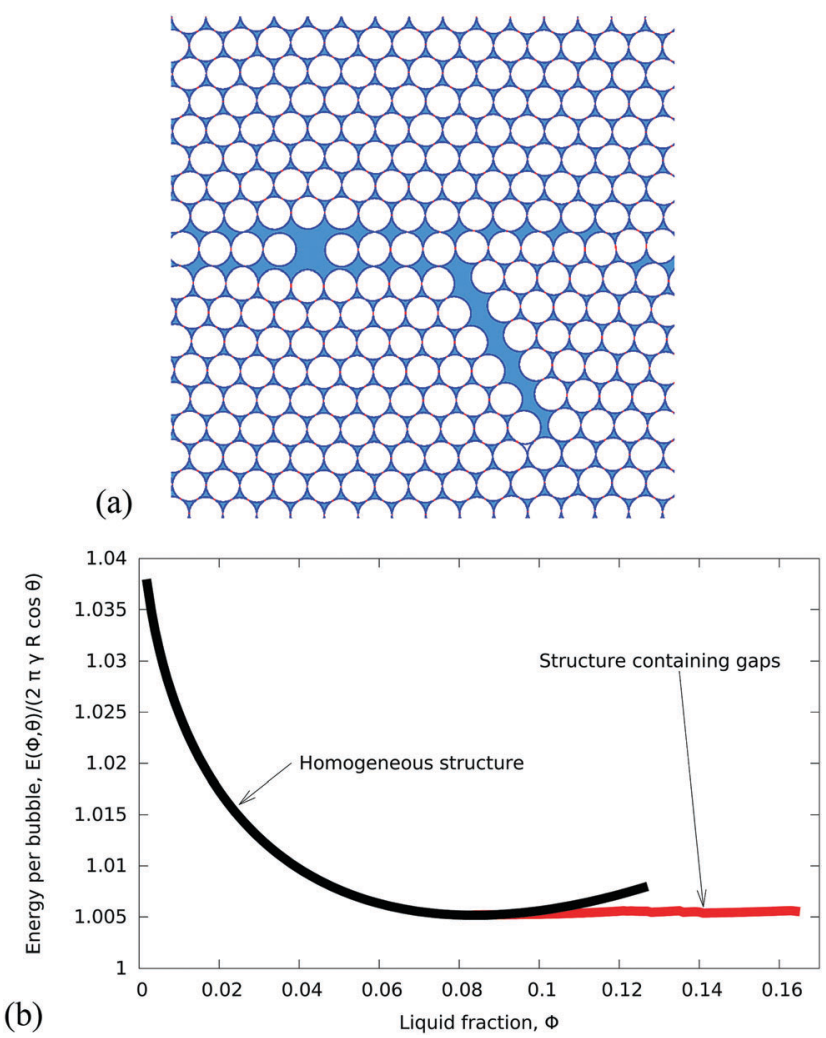

Fig. 7 (a) Example of a Surface Evolver simulation of a monodisperse foam ( $\phi=0.12$, contact angle $\theta=6.26^{\circ}, 256$ bubbles) which shows the formation of inhomogeneities upon an increase in liquid fraction above $\phi_{0}^{\theta}=0.084$. The appearance of inhomogeneities was triggered by a random perturbation of the ordered structure. (b) Upon a further increase in liquid fraction the energy remains roughly constant.

The inhomogeneity does not arise spontaneously in these simulations, but requires a perturbation at a liquid fraction just above $\phi_{0}^{\theta}$. This is achieved by randomly displacing all of the Plateau border vertices by a small distance of about $0.13 R$; this allows the system to escape from the metastable branch by undergoing topological transitions that are triggered when film lengths go to zero. These result in the formation of cracks, or liquid pools, as illustrated in Fig. 7. The requirement of a perturbation to trigger instability is a familiar feature of highly symmetric structures that are locally stable, for which alternative structures of lower energy are available. Subsequently, increasing or decreasing the liquid fraction keeps the foam at roughly constant energy. Small fluctuations in the energy occur because, as the liquid fraction changes in this finite sample of foam, there are short periods during which the energy increases elastically, followed by topological transitions that reduce the energy. As the sample size increases, such fluctuations become less marked.

This instability is reminiscent of the observations by Abd el Kadar and Earnshaw ${ }^{16}$ in experiments with monodisperse 2D bubble rafts in an hexagonal confinement. About 25 minutes after foam formation, cracks appeared within the perfectly ordered structure, rapidly leading to the formation of a hole in the monolayer. No bubbles are lost in this process. The authors attribute the development of this inhomogeneity to the movement and build-up of local stresses, for example due to small variations in bubble size. It is conceivable that the existence of a finite contact angle plays a further role, although the concept cannot be applied straightforwardly to bubble rafts, as opposed to $2 \mathrm{D}$ foam trapped between plates.

\section{Disordered foams with finite contact angle}

Having established the effect of a finite contact angle for ordered foams we now turn to the results of Surface Evolver simulations of disordered foams, as exemplified in Fig. 8. The results presented below are based on five samples with $N=1500$ bubbles and different values of the contact angle $\theta$ between $2.6^{\circ}$ and $15.9^{\circ}$. The foams are polydisperse: the bubbles have average area close to 1 but the variation in bubble areas differs between samples. The polydispersity is captured by the parameter

$$
p=\frac{R_{21}}{\left\langle R^{2}\right\rangle^{1 / 2}}-1,
$$

where $R_{21}=\frac{\left\langle R^{2}\right\rangle}{\langle R\rangle}$ is the Sauter mean radius in $2 \mathrm{D}$ and the average, denoted with \langle\rangle , is over all bubbles in the foam. Details of the simulations are presented in Appendix A. A video of the wetting of a foam with contact angle $15.9^{\circ}$ is available in the ESI. $\dagger$

We simulate the wetting of a dry foam by increasing the area of liquid and keeping the area of gas constant. We again find that inhomogeneities develop as the liquid fraction $\phi$ is increased. However, these inhomogeneities do not necessarily result from metastable structures of the kind discussed in the previous section. The disordered samples spontaneously undergo discrete topological transitions as the liquid fraction is increased; these occur when the length of a film between two neighbouring bubbles goes to zero, causing them to separate. In the simplest case, two bubbles that share a shrinking edge separate, and two three-sided Plateau borders merge to form a four-sided Plateau border, as shown in the Appendix, Fig. 11. Similarly, non-adjacent edges on a Plateau border with four or more sides may come into contact, and a new edge is formed. Various combinations of these topological transitions are responsible for the highly irregular liquid regions shown in Fig. 8.

Fig. 9 illustrates the growth of inhomogeneity as the liquid fraction is increased. The evolving inhomogeneity exhibits an acceleration in the growth of a few, increasingly larger, Plateau borders, while most remain small. The area of the largest Plateau border, $A_{\mathrm{pb}}^{\max }$, within a sample (Fig. 9) increases roughly exponentially with liquid fraction.

The average area of the Plateau borders $\left\langle A_{\mathrm{pb}}\right\rangle$ is given by

$$
\left\langle A_{\mathrm{pb}}\right\rangle=\left(\frac{2 N}{N_{\mathrm{pb}}}\right) \frac{\langle A\rangle}{2} \frac{\phi}{1-\phi},
$$

where $N$ is the constant number of bubbles, $\langle A\rangle$ is the constant average bubble area, and $N_{\mathrm{pb}}$ is the number of Plateau borders, 

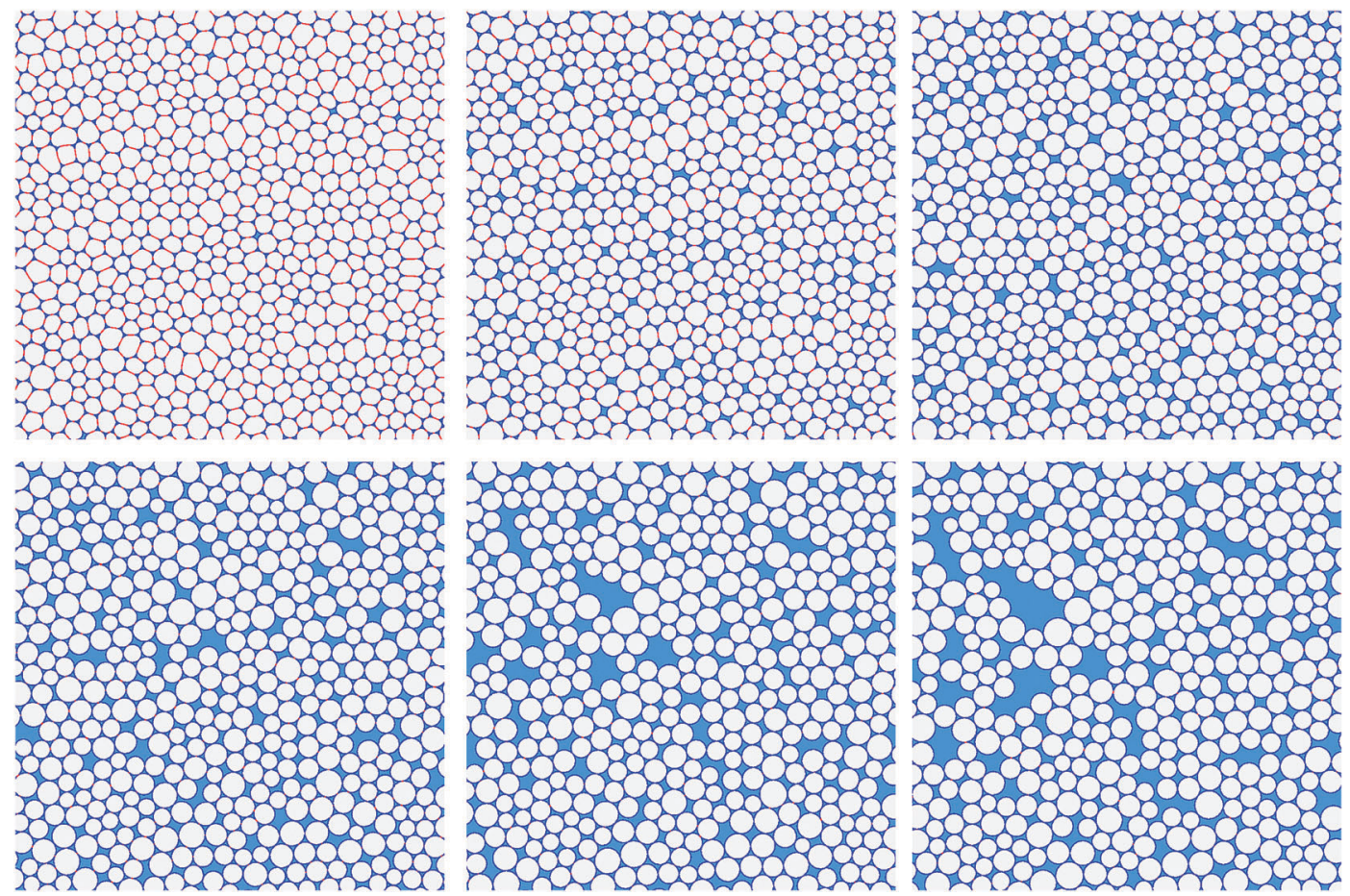

Fig. 8 Surface Evolver simulation of the equilibrium structure of a disordered foam of 1500 bubbles with average area equal to one, polydispersity $p=0.0086$, and contact angle $\theta=2.6^{\circ}$. An increase in liquid fraction leads to the appearance of cracks or incipient flocculation. The images shown correspond to close-ups of the foam at values of liquid fraction $\phi=0.04,0.08,0.12,0.16,0.20$ and 0.24 (from top left to bottom right).

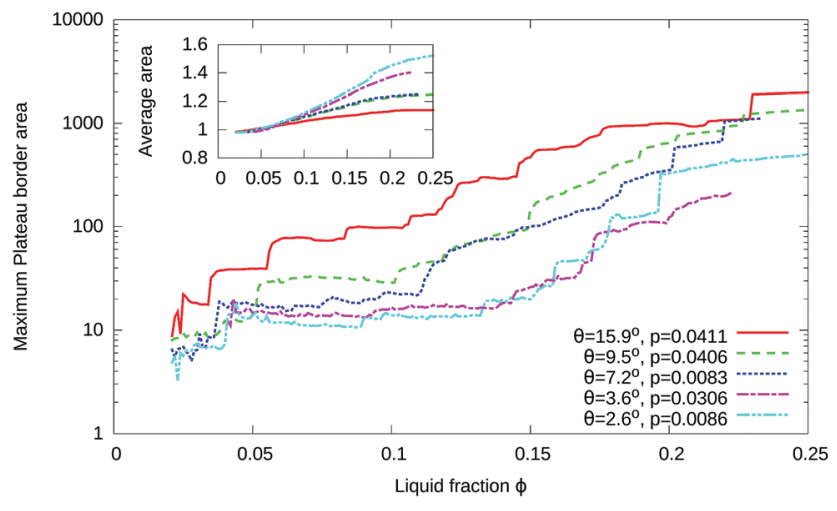

Fig. 9 The development of inhomogeneities in disordered foams with finite contact angle is illustrated by the observation that, while the average area of a Plateau border $\left\langle A_{\mathrm{pb}}\right\rangle$ grows sublinearly with liquid fraction $\phi$ (inset), the area of the largest Plateau border $A_{\mathrm{pb}}^{\max }$ (at each value of $\phi$ ) grows approximately exponentially (note the logarithmic vertical axis). The data shown is for five simulations of disordered foams, with values of contact angle as indicated. The areas are normalized by the average area $\left\langle A_{\mathrm{pb}}\right\rangle$ that would result if Plateau borders did not merge, eqn (9). Note that the drops in area at low liquid fraction correspond to the splitting of a Plateau border with more than three sides into three-sided Plateau borders, as explained in Appendix A.

which decreases when they merge. If the Plateau borders do not merge, i.e. if they all remain three-sided, the pre-factor $2 N / N_{\mathrm{pb}}$ is unity. The average Plateau border area, scaled by the value of $\left\langle A_{\mathrm{pb}}\right\rangle$ that would result if they didn't merge (and remained three-sided, as they were when the foam was created at low liquid fraction), is shown in the inset to Fig. 9. Note that the average Plateau border area rises more quickly at lower contact angles; this occurs because the tendency to combine is more pronounced when the contact angle is smaller, so the film lengths are smaller, and consequently, topological transitions are more frequent.

The energy per bubble, defined as the total energy divided by the number of bubbles $N$, is plotted as a function of liquid fraction in Fig. 10. It has been rescaled by $(1+p)$ to compensate for the polydispersity of the samples, as explained in Appendix B. As in the case of ordered foams, an increase in contact angle

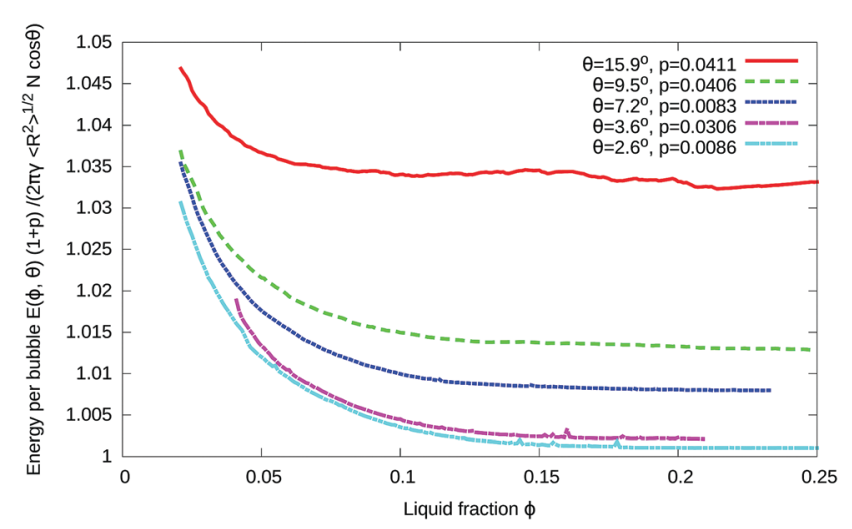

Fig. 10 Variation of the energy with liquid fraction $\phi$ for five disordered foams with finite contact angles. The gradual development of inhomogeneities, as shown in Fig. 8, is accompanied by a decrease in energy as a function of $\phi$ followed by saturation to a roughly constant value. The energy has been rescaled using the polydispersity parameter $p$ defined in eqn (8). 
leads to an increase in energy for a given value of the liquid fraction. For fixed contact angle, the energy decreases with increasing liquid fraction, at first steeply, and then more slowly. The initially monotonic decrease in energy with $\phi$ is consistent with the gradual development of inhomogeneities that we observe in disordered foams.

What should we expect the eventual state of a foam to be, were this wetting process to be continued to ever higher values of liquid fraction, beyond the usual 2D wet foam limit? We expect that the presence of a finite contact angle, which causes an effective adhesion between the bubbles, will lead to one large cluster of bubbles surrounded by liquid. This is again reminiscent of the clustering seen in $2 \mathrm{D}$ bubble rafts. ${ }^{18}$

\section{Summary and conclusions}

Due to their increased stability, adhesive or "sticky" 3D emulsions, featuring droplet flocculation, ${ }^{13,19,20}$ find many applications in the food and cosmetics industries. Here we have discussed flocculation in the case of the analogous system of liquid foams.

Unlike the familiar case of a foam with zero contact angle, an ideal two-dimensional foam with a finite contact angle develops an inhomogeneity for high liquid fraction $\phi$. In an ordered foam this inhomogeneity appears at a critical value of $\phi$, but in a disordered foam there is a gradual development of inhomogeneity with increasing $\phi$. This implies that the notion of the wet limit is ill-defined in foams with finite contact angle.

As in other aspects of foam physics, our results for 2D foam should give a general indication of the corresponding properties of $3 \mathrm{D}$ foams and emulsions. It is surprising that the subject of finite contact angles has remained underdeveloped, and it is hoped that the present results will stimulate further experiments and simulations. Indeed, there does not appear to be any $2 \mathrm{D}$ data that could be compared to our findings.

In light of the described inhomogeneous structures, it may now be of interest to revisit earlier work on the ideal disordered 2D foam model, but including a finite contact angle. For example, does a finite contact angle affect the statistics of bubble rearrangements, perhaps by suppressing system-wide avalanches of topological changes, following a small increase in $\phi ?^{21}$

\section{Conflicts of interest}

There are no conflicts to declare.

\section{Appendix A. Simulations using the Surface Evolver}

We carried out simulations of both ordered (hexagonal) and disordered (polydisperse) foams with finite contact angles using Ken Brakke's Surface Evolver software ${ }^{10}$ to relax an initial foam structure to a minimum of the surface energy (which is proportional to the total perimeter), subject to fixed bubble areas.

In principle, all the properties of an ordered hexagonal foam can be captured from a single cell, considered with periodic boundary conditions. However, since we seek an instability of this structure to an inhomogeneous state, we must instead simulate many cells. We chose to reproduce the basic hexagonal cell 256 times to form a $16 \times 16$ hexagonal lattice with periodic boundary conditions.

The disordered foams are made as dry foams, also with periodic boundary conditions, from a Voronoi construction in the usual way; ${ }^{22}$ these have $N=1500$ bubbles, with average area $\langle A\rangle$ close to 1 , and different polydispersity.

Both ordered and disordered foams are turned into wet foams of liquid fraction $\phi \approx 0.03$ by adding a small triangular Plateau border at each three-fold vertex; the liquid fraction is set by the total area of all Plateau borders. They have no individual area constraints, and therefore all have the same pressure.

Each side of each Plateau border is associated with a fixed bulk interfacial tension $\gamma \geq \frac{1}{2}$; the interfacial tension in the thin liquid films is set to $\gamma_{\mathrm{f}}=1$. The contact angle is then given by eqn (1). The current version of the Surface Evolver software does not allow for the simulation of $2 \mathrm{D}$ foams with zero contact angle and we find that $\theta=2.6^{\circ}$ is the minimum value that we can choose to ensure convergence. Examination of the consequences of this limitation provided some initial motivation for this study.

In the Surface Evolver, each edge is represented as a circular arc, and a local minimum of the interfacial energy is sought using up to $2 \times 10^{4}$ iterations to achieve a relative accuracy close to $10^{-6}$.

The liquid fraction is changed by increasing the area of liquid and keeping the area of gas constant (so the size of the periodic box increases). Small increments in $\phi$ allow us to explore a large range of liquid fractions: at each step, the liquid fraction is increased by 0.001 , up to about $\phi=0.25$. We used the gradient descent method for energy minimisation, with occasional Hessian iterations, to move towards a minimum of interfacial energy $\sum_{j} \gamma_{j} L_{j}$. Here $L_{j}$ denotes the lengths of the interfaces, and $\gamma_{j}$ is either $\gamma$ or $\gamma_{\mathrm{f}}$, depending on whether the interface is associated with a Plateau border or a film.

As the foam evolves, bubble rearrangements (topological changes) occur. We chose a critical film length of $10^{-4}$ below which these are triggered. The difficult step is in recognising when a four-sided Plateau border should split into two threesided Plateau borders (or, in general, a many-sided Plateau border should split into two parts). To achieve this we check whether two sides of any Plateau border with more than three sides overlap, as illustrated in Fig. 11. If they do, these two sides are joined, to split the Plateau border. We briefly increase $\gamma$ and perform a few iterations, and then the minimization continues.

\section{Appendix B. Energy of a polydisperse 2D foam}

The energy of a foam decreases with increasing polydispersity in the bubble areas at fixed liquid fraction. ${ }^{17}$ We capture this 
(a)

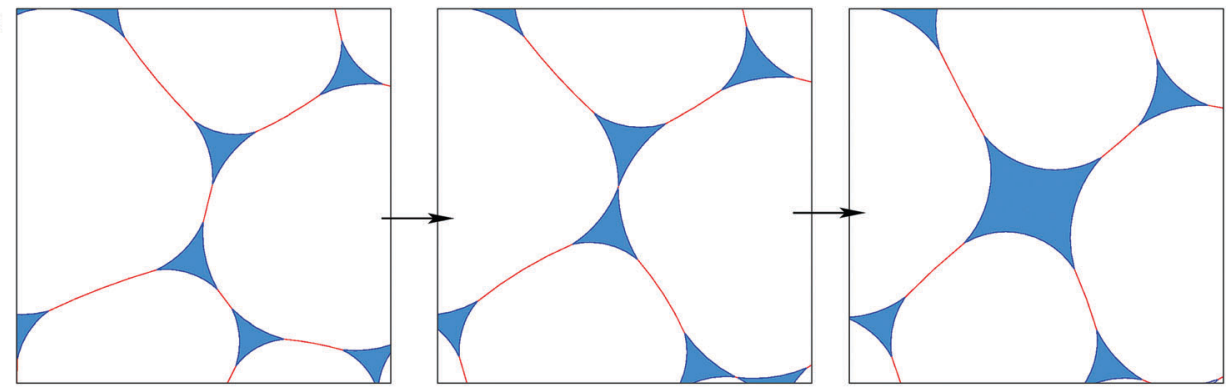

(b)

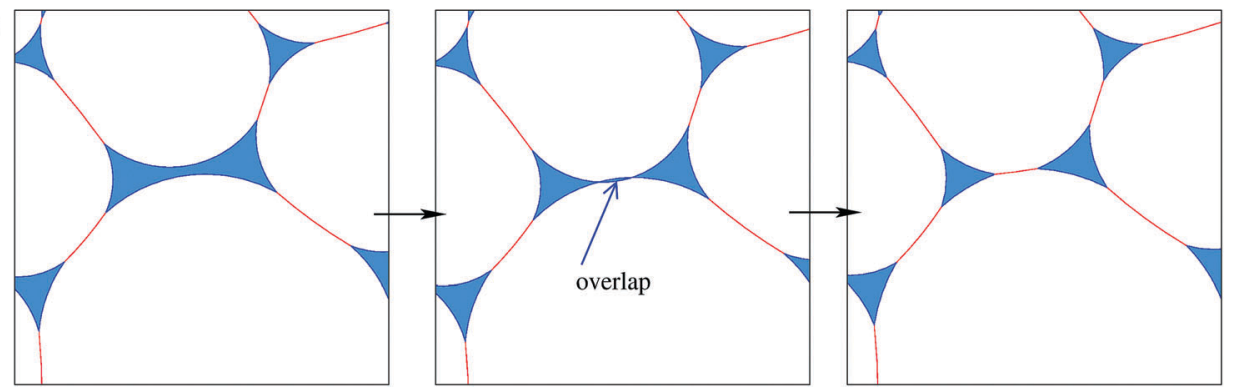

Fig. 11 Numerical method for performing topological changes. (a) Two three-sided Plateau borders join when the interface between them shrinks below a critical length. The four-sided Plateau border is created by deleting this short interface and merging the Plateau borders. (b) Two sides of a foursided Plateau border approach each other; when they overlap, a new interface is inserted, creating two three-sided Plateau borders. A similar process is used for Plateau borders with more than four sides. Note that all Plateau borders have the same pressure, irrespective of their number of sides, and that their total area is conserved (rather than individual areas), so that no repartition of the liquid is required.

dependence as follows. We define polydispersity in terms of the $2 \mathrm{D}$ Sauter mean radius according to eqn (8) and then the energy of an equilibrium, dry (i.e., $\phi=0$ ), 2D foam can be written as

$$
E=\frac{c}{1+p} 2 \pi \gamma\left\langle R^{2}\right\rangle^{1 / 2} N
$$

The parameter $c$ is estimated to be $1.056 \pm 0.007$, where the error value indicates the weak dependence on bubble shape, as in $3 \mathrm{D} .{ }^{17}$ In plotting Fig. 10 we have assumed that the same scaling of $(1+p)^{-1}$ applies at finite values of the liquid fraction $\phi$.

\section{Acknowledgements}

SJC wishes to thank K. Brakke for advice about Surface Evolver simulations and R. Höhler for useful discussions. SH and DW acknowledge the support of the MPNS COST Actions MP1106 Smart and green interfaces and MP1305 Flowing matter. The visit of AMK to the TCD Foams and Complex Systems group was funded by the TCD Visiting Professors Fund and the Dublin Graduate Physics Program, and his visit to Aberystwyth was funded by the Distinguished Visiting Fellowship Scheme of the Royal Academy of Engineering. Financial support from Science Foundation Ireland (SFI) under grant number 13/IA/1926 (SH, DW) and the European Space Agency ESA, Project microG-Foam, AO99075 and contract 4000115113, 'Soft Matter Dynamics' (SJC, SH, DW) is also gratefully acknowledged.

\section{References}

1 F. Bolton and D. Weaire, The effects of Plateau borders in the two-dimensional soap froth. II. General simulation and analysis of rigidity loss transition, Philos. Mag. B, 1992, 65, 473-487.

2 Z. Jing, S. Wang, M. Lv, Z. Wanga and Z. Luoa, The effect of plastic rearrangements on the flow of two-dimensional wet foam, Soft Matter, 2015, 11, 2973-2982.

3 C. S. Smith, The shapes of metal grains, with some other metallurgical applications of topology, Metal Interfaces, ASM, Cleveland, 1952, pp. 65-108.

4 S. J. Cox and E. Janiaud, On the structure of quasi-twodimensional foams, Philos. Mag. Lett., 2008, 88, 693-701.

5 D. Weaire and S. Hutzler, The Physics of Foams, Oxford University Press, 1999.

6 I. Cantat, S. Cohen-Addad, F. Elias, F. Graner, R. Höhler, O. Pitois, F. Rouyer and A. Saint-Jalmes, Foams: Structure and Dynamics, Oxford University Press, 2013.

7 H. M. Princen, Highly concentrated emulsions. I. Cylindrical systems, J. Colloid Interface Sci., 1979, 71(1), 55-66.

8 D. Bonn, J. Eggers, J. Indekeu, J. Meunier and E. Rolley, Wetting and spreading, Rev. Mod. Phys., 2009, 81, 739.

9 D. Langevin, Surfactants, in The Oxford Handbook of Soft Condensed Matter, ed. E. M. Terentjev and D. A. Weitz, Oxford University Press, USA, 2015, pp. 51-93.

10 K. A. Brakke, The Surface Evolver, Exp. Math., 1992, 1, 141-165. Software free to download from http://facstaff. susqu.edu/brakke/evolver/evolver.html. 
11 H. M. Princen and S. G. Mason, Shape of a fluid drop at a fluid-liquid interface. I. Extension and test of two-phase theory, J. Colloid Interface Sci., 1965, 20(2), 156-172.

12 H. M. Princen, Contact angles and transition regions in soap films, J. Phys. Chem., 1968, 72(9), 3342-3345.

13 R. Aveyard, B. P. Binks, J. Esquena, P. D. I. Fletcher, R. Buscall and S. Davies, Flocculation of weakly charged oil-water emulsions, Langmuir, 1999, 15(4), 970-980.

14 H. M. Princen, Osmotic pressure of foams and highly concentrated emulsions. I. Theoretical considerations, Langmuir, 1986, 2(4), 519-524.

15 S. Hutzler and D. Weaire, The osmotic pressure of a twodimensional disordered foam, J. Phys.: Condens. Matter, 1995, 7(47), L657.

16 A. Abd el Kader and J. C. Earnshaw, Stability of twodimensional foam, Philos. Mag. A, 1997, 76(6), 1251-1260.
17 A. M. Kraynik, D. A. Reinelt and F. van Swol, Structure of random foam, Phys. Rev. Lett., 2004, 93, 208301.

18 C. Kuo, A. Levine and M. Dennin, Bubble Ribbons under Imposed Flow, Colloids Surf., A, 2017, 534, 38-43.

19 J. Bibette, T. G. Mason, H. Gang, D. A. Weitz and P. Poulin, Structure of adhesive emulsions, Langmuir, 1993, 9(12), 3352-3356.

20 R. Aveyard, B. P. Binks, J. Esquena, P. D. I. Fletcher, P. Bault and P. Villa, Flocculation transitions of weakly charged oil-inwater emulsions stabilized by different surfactants, Langmuir, 2002, 18(9), 3487-3494.

21 F. F. Dunne, F. Bolton, D. Weaire and S. Hutzler, Statistics and topological changes in $2 \mathrm{~d}$ foam from the dry to the wet limit, Philos. Mag., 2017, 97, 1768-1781.

22 K. A. Brakke, Statistics of non-Poisson point processes in several dimensions, 2006, http://facstaff.susqu.edu/brakke/ aux/downloads/papers/nonpoisson.pdf. 